Revista Internacional de

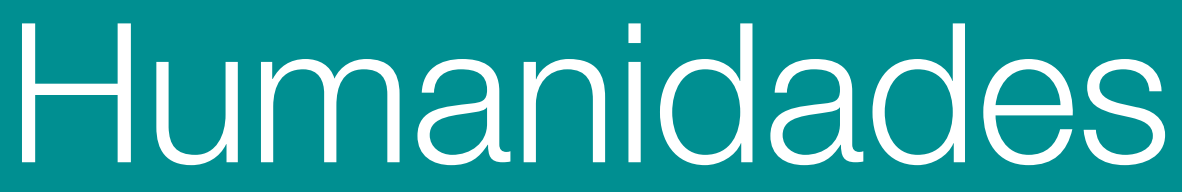

Profesores adolescentes

Herramienta didáctica para Lengua y Literatura Española 


\section{REVISTA INTERNACIONAL DE HUMANIDADES}

Primera Edición Common Ground Research Networks 2021

University of Illinois Research Park

2001 South First Street, Suite 202

Champaign, IL 61820 USA

Tel.: +1-217-328-0405

www.cgespanol.org

ISSN: 2474-5022 (versión impresa)

ISSN: 2253-6825 (versión electrónica)

(C) 2021 (artículos individuales), autor(es)

(C) 2021 (selección y contenido editorial), Common Ground Research Networks

Todos los derechos reservados. Excepto propósitos de estudio, investigación, crítica o revisión permitidos bajo la legislación de

derechos de autor, ninguna parte de este trabajo puede ser reproducida,

en ningún formato, sin el consentimiento explícito por escrito del editor.

Para otros tipos de permisos y dudas, por favor, escriba a:

soporte@cgespanol.org.

La Revista Internacional de Humanidades

es una publicación académica arbitrada bajo el proceso de revisión por pares. 


\title{
Profesores adolescentes: herramienta didáctica para Lengua y Literatura Española
}

\author{
(Teenage teachers: didactic tool for Spanish Language and Literature)
}

María Pareja-Olcina, ${ }^{1}$ Grupo de investigación Talis, Universitat Jaume I, España

\begin{abstract}
Resumen: El aula de secundaria es un espacio abierto al discurso lingüístico. Por tanto, el desarrollo de estas habilidades resulta esencial en la vida escolar para: aprender a aprender, explorar el lado más creativo del lenguaje a partir de la literatura, cuestionar y reflexionar sobre las propias ideas y las que recibimos de los otros. Ante este paradigma, realizamos una propuesta de mejora educativa, a través de un estudio de caso, con el fin de activar la motivación, integrar el aprendizaje sobre la literatura del alumnado de $1^{o}$ de Bachillerato y mejorar sus destrezas comunicativas orales. En el estudio participaron tres grupos mixtos de un centro público (68 estudiantes en total). Los resultados de esta actividad discursiva evidencian que el estudiante de estos niveles educativos (16 o 17 años) integra mejor las habilidades lingüísticas y curriculares si le permitimos convertirse en ponente. El trabajo que hemos realizado es una invitación para que el profesorado acepte el potencial educativo de ceder espacio y tiempo en el aula al estudiante para que se convierta en comunicador y protagonista de su propio aprendizaje.
\end{abstract}

\section{Palabras clave: pedagogía, lengua y literatura española, bachillerato, discurso lingüístico}

\begin{abstract}
The high school classroom is a space open to linguistic speech. Therefore, the development of these skills is essential in school life to: learn to learn, explore the most creative side of language from literature, question and reflect on one's own ideas and those we receive from others. Given this paradigm, we generated a proposal for educational improvement in order to activate motivation, integrate learning about the literature selection from the first course of Bachillerato and improve their oral communication skills. Our case study presents the implementation of this proposal with three mixed groups from a public school (68 students in total). The results of this discursive activity show that students in these educational levels (16 or 17 years old) integrate linguistic and curricular skills more efficiently if they are allowed to become speakers. The case study intends to be an invitation for teachers to integrate the educational potential of giving space and time in the classroom for the student to become a communicator and the protagonist of their own learning.
\end{abstract}

Keywords: Pedagogy, Spanish Language and Literature, High School, Linguistic Speech

\section{Introducción}

$\mathrm{V}$

ivimos en una era digital, en la que debemos educar para aceptar el cambio y la formación constante. En estas últimas décadas se ha investigado mucho sobre aprendizaje autodirigido para conseguir esa continuidad, lifelong learning (Candy 1991). El término lifelong learning o aprendizaje a lo largo de la vida, comprende todas las actividades de aprendizaje en la trayectoria educativa de una persona con el objetivo de aumentar el conocimiento y mejorar sus competencias personales, lejos ha quedado el concepto ilustrado que entendía que en la vida del ser humano hay una edad destinada a la instrucción y otra a desempeñar una tarea en función de lo aprendido (Jovellanos 2012). La realidad actual, para funcionar en el mundo del siglo XXI, nos empuja a una mejora personal continuada, a un aprendizaje constante, así que aprender a aprender nos resulta más interesante que qué aprendemos, porque entendemos que el estudiante del presente lo necesitará para su futuro

\footnotetext{
${ }^{1}$ Corresponding Author: María Pareja Olcina: Av. Vicent Sos Baynat, s/n, Department European Philology and Cultures - Spanish Language, Associate professor, Castellón, Comunidad Valenciana,12071, Spain, email: pareja@uji.es
}

Revista Internacional de Humanidades

Volumen 8, Número 2, 2021, https://las-humanidades.com/revistas.

(C) María Pareja Olcina.

Publicado por Common Ground Research Networks.

Attribution License, (CC BY 4.0).

ISSN: 2474-5022 (versión impresa), ISSN: 2253-6825 (versión electrónica)

http://doi.org/10.18848/2474-5022/CGP/v08i02/19-36 (Article) 
personal y laboral. La publicación de un tratado teórico-práctico de enseñanza, con aplicación en escuelas y colegios por Gaspar Melchor de Jovellanos, en 1801, supuso un punto de inflexión educativa para lograr una renovación pedagógica abierta, libre y adecuada a las clases sociales. Hoy en día, el reto educativo pasa por replantearnos cómo aprendemos y en darle protagonismo al estudiante, de forma que el profesor se convierta en un mediador metodológico y no en un suministrador de contenidos (Osses y Jaramillo 2008). Así, un punto esencial recogido en el estudio de Philip Candy (1991) se basa en un concepto constructivista, puesto que el estudiante construye estructuras cognitivas idiosincrásicas relacionadas con su experiencia previa (Candy: 270) que enfatizan y consolidan el aprendizaje. Estas estrategias aparecen recogidas en las Reformas Educativas de Europa y más concretamente en España, que en su última actualización del sistema educativo (LOMLOE del 13 de marzo de 2020), considera uno de sus objetivos principales modernizar el sistema educativo, centrándose en el alumnado y su potencial a desarrollar ${ }^{2}$. En esta línea, el Real Decreto sobre el currículo básico de la Educación Secundaria Obligatoria y del Bachillerato (1105/2014, de 26 de diciembre), establece en el artículo 29 que "las actividades educativas en el Bachillerato favorecerán la capacidad del alumnado para aprender por sí mismo, para trabajar en equipo y para aplicar los métodos de investigación apropiados" y que "las Administraciones educativas promoverán las medidas necesarias para que [...] se desarrollen actividades que estimulen el interés y el hábito de la lectura y la capacidad de expresarse correctamente en público".

Una de las nuevas prioridades de la organización del currículo de Lengua Castellana y Literatura atiende a la formación en el dominio de todos los procesos y habilidades lingüísticocomunicativas. El objetivo que se persigue en la LOE es el desarrollo de la competencia comunicativa del alumnado, lo que implica conocer el código, pero también saber qué y cómo decirlo según la situación comunicativa. Y para ello se aplicarán los recursos y las metodologías necesarias para desarrollar la competencia comunicativa y literaria, de forma que el estudiante avance en el proceso de integración social y supere los retos laborales del siglo XXI.

En el marco europeo hay un interés por abordar el aspecto educativo, considerando el papel fundamental que desempeñan la educación, la juventud y la cultura en la construcción del futuro de Europa. En este sentido, el 30 de septiembre de 2020 se publicó una Comunicación sobre el Espacio Europeo de Educación, en la que se define el recorrido que deben seguir los países miembros para lograr avances educativos en 2025 (European Commission 2020). El paquete de medidas políticas en el se está trabajando desde Europa busca aprovechar al máximo el potencial de la educación y la cultura como motores de creación de empleo, crecimiento económico y mejora de la cohesión social, y como medio para experimentar la identidad europea en toda su diversidad.

En vista de las demandas sociales, nuestro objetivo como profesores es enseñar a los estudiantes la mejor forma de aprender para que puedan aumentar su autonomía y aplicar esta técnica a lo largo de su vida. En este sentido, las exposiciones orales se han convertido en una herramienta esencial para integrar el contenido académico en el área de Lengua y Literatura Española (Gràcia et al. 2017). En el aula, hemos visto una diferencia sustancial entre realizar exámenes de contenido memorístico, que el alumno olvida una vez ha finalizado la prueba, y darle la posibilidad de convertirse en docente (McGuire 2020). En este último caso, el estudiante investiga, lee, se prepara oralmente, reflexiona sobre la mejor manera de explicar a sus compañeros el contenido y realiza su presentación oral. Finalmente, resuelve las dudas que hayan podido surgir de esta, por eso es importante que domine el contenido que prepara.

2 Proyecto de Ley orgánica de modificación de la LOE (LOMLOE). Rescatado el 18 de diciembre de 2020: https://www.educacionyfp.gob.es/dam/jcr:f19685f1-0260-4926-b043-768d82ed09e0/bocg-d-14-113-983senado.pdf 


\section{Marco teórico}

Las investigaciones de la última década sobre el aprendizaje (Alonso y Gallego 2003; Dehaene 2020; Jandrić et al. 2020; Kennedy 2019; Navarro 2008; Salmerón, et al. 2009, 2010, 2011) han hecho hincapié en cómo aprendemos y entienden que cada estudiante aprende de forma diferente según el contexto, estrategias, edad, motivación, aprendizaje o conocimientos previos. Los investigadores han intentado ofrecer un concepto sobre cómo aprendemos, pero estos conceptos están llenos de matices y elementos que debemos considerar. Así, para Gregorc (1979) el aprendizaje tiene una relación directa con comportamientos distintivos que sirven como indicadores de cómo aprende una persona y se adapta a su entorno. Para ello nos presenta cuatro descriptores (1982) que nos permitirían reconocer varios tipos de estudiantes:

- Alumno secuencial concreto: ordenado, perfeccionista y práctico.

- Alumno secuencial abstracto: lógico, analítico, racional y evaluativo.

- Alumno abstracto aleatorio: sensible, colorido, emocional y espontáneo.

- Alumno aleatorio concreto: intuitivo, independiente, impulsiva y original.

Según Gregorc el estudiante puede hacer uso de forma indistinta de estos tipos, pero hay inclinaciones innatas hacia uno o dos de ellos (2002) y cada tipo tiene atributos potencialmente positivos y negativos. Otros autores, (Esteban et al. 1996; Schmeck, 1982; Shi 2017) entienden el estilo de aprendizaje como estrategias particulares que el individuo manifiesta según su tarea académica y Hong Shi (2017) anima a los profesores a elegir técnicas de enseñanza y aprendizaje adecuadas para los estudiantes. Para Kolb (1984) estas capacidades de aprender están relacionadas con factores hereditarios, experiencias vitales o del medio ambiente en el que se desarrollan. Según Alonso et al. (1997), Hewitt et al. (2008) y Keefe (1988) estos estilos tienen que ver con los rasgos cognitivos, afectivos y fisiológicos que sirven como indicadores de cómo los estudiantes perciben, interaccionan y responden en sus ambientes de aprendizaje. En esta línea, Dunlosky et al. (2013) y Hunt (1979) destacan las condiciones educativas en las que se encuentra el alumno para aprender mejor. Este trabajo ha encontrado en esta tendencia un punto de partida, basada en quince años de experiencia en Secundaria, en la que a través de la observación hemos comprobado que la inteligencia heredada no constituye un factor determinante, a no ser que venga condicionada por otros factores. En este sentido, nuestro cometido estaría directamente vinculado en ofrecer las condiciones educativas necesarias a nuestro alumnado para lograr los indicadores de éxito académico. Es decir, la genética es un rasgo físico que ayuda al aprendizaje, pero esta formación viene determinada por habilidades que se pueden mejorar a través de la experiencia y en las que el interés y la motivación constituyen claves esenciales en este proceso (Entwistle 1988; Renninger 2015).

En cuanto a estrategias de aprendizaje, Fernández (2004, 412) las define como las "operaciones mentales, mecanismos, técnicas, procedimientos, planes, acciones concretas que se llevan a cabo de forma potencialmente consciente y que movilizan los recursos para maximizar la eficacia tanto en el aprendizaje como en la comunicación". En este apartado también encontramos diferentes posturas. Chow et al. (2011) y Weinstein et al. (1985) identifican una serie de habilidades útiles para que el aprendizaje sea efectivo: retención de la información, comprensión y aplicación posterior de esa información, el grupo dirigido por Alan Chow (2011) añade a estos conceptos el valor de incorporar actividades alternativas (juegos, ejercicios y simulaciones) para estimular el interés de los estudiantes, mejorar la transferencia de conocimientos y la retención de lo aprendido con la repetición significativa. Asimismo, Schmeck (1988) señala como estrategia la predisposición del estudiante, con independencia de la tarea que vaya a realizar. Mientras que para Derry y Murphy (1986) estas estrategias constituyen un conjunto de actividades mentales que utiliza el estudiante en una situación particular, para facilitar la adquisición de conocimiento. Al respecto, encontramos estudios (Castejón, Montanes y García 1993; Monereo y Castelló 1997) que señalan que el ámbito de 
análisis que hemos seleccionado, Secundaria, se ve afectado por las estrategias metacognitivas de atención, comprensión y memoria. Creemos que nuestra propuesta ayuda al estudiante a focalizar el control de la atención sobre su exposición oral, la comprensión significativa de los contenidos de aprendizaje y la necesidad de recordarlos. A modo de recapitulación citamos la clasificación de estrategias de Fernández $(2004,423)$, que a su vez están basadas en el modelo de Oxford (1990):

- Estrategias de aprendizaje directas: memoria (necesarias en la presentación oral), cognitivas (sacar la idea principal, utilizar recursos variados para comunicar, analizar y razonar el contenido que presentan) y compensatorias (superar limitaciones y carencias en la lengua hablada a través de claves lingüísticas y extralingüísticas: gestos, utilizar sinónimos, adaptar el lenguaje a lo que se conoce...).

- Estrategias de aprendizaje indirectas: metacognitivas (organización, planificación y autoevaluación del propio trabajo antes de su presentación), afectivas (técnicas de relajación y control emocional ante la exposición de un tema a un público), sociales (cooperar con su grupo, resolver dudas, intentar comprender el momento histórico, la cultura, los pensamientos y sentimientos de escritores de otras generaciones...)

Las posibilidades educativas de incorporar esta dinámica de trabajo en las aulas son múltiples y, por esta razón, este estudio surge de la necesidad de dar respuesta a una inquietud entre el profesorado de educación secundaria: ¿cómo conseguir que los adolescentes integren las propuestas clave del currículum de forma amena y eficaz?

\section{Metodología}

Este trabajo utiliza un método de evaluación cualitativa a través del estudio de caso (Stake 1998), en el marco de la investigación-acción educativa en la construcción de saber pedagógico por parte del docente investigador, con el objetivo de encontrar un sistema de enseñanza que mejore el rendimiento académico y la motivación del alumnado (Walker 2002). Para recoger los datos nos hemos basado en los resultados académicos, encuestas y una entrevista oral a cada grupo.

El proyecto se aplicó en la asignatura de Lengua y Literatura Española en un centro público de la Comunidad Valenciana (España), IES Violant de Casalduch, a lo largo del primer trimestre durante el curso 2020-2021. Los participantes han sido seleccionados de forma no aleatoria de tres grupos mixtos de $1^{\circ}$ de Bachiller, el número total ha sido de 68 estudiantes con edades comprendidas entre los 16-17 años. Para realizar la actividad establecimos un día fijo semanal. Es decir, de las tres sesiones de Lengua Castellana y Literatura que se tienen a la semana escogimos una sesión con cada grupo para realizar la adaptación metodológica. La elección del día tampoco fue al azar ya que, dentro del horario, buscamos la hora que se encontraba en una franja inferior de la jornada escolar, lo hicimos así porque el rendimiento del estudiante es menor durante las últimas horas y con esta dinámica se conseguía mantener su atención. Las franjas escogidas fueron las siguientes:

- $1^{\circ}$ Bachiller B: lunes de 13.15 a 14.10

- $\quad 1^{\circ}$ Bachiller C: jueves de 12.20 a 13.15

- $\quad 1^{\circ}$ Bachiller D: viernes de 13.15 a 14.10

El alumnado que nos encontramos en este nivel, durante este curso, tienen unas características peculiares que los diferencian de otros grupos de Bachiller de años anteriores, esto se debe a las condiciones del curso anterior. El confinamiento por COVID-19 supuso que un número significativo de estos estudiantes aprobara sin integrar unos mínimos curriculares y unos hábitos de trabajo. Algunos de ellos confiesan que su intención era reconducir su carrera académica hacia la Formación Profesional, pero que la nota de corte que obtuvieron fue insuficiente y por esta razón se matricularon en Bachiller. En este sentido, hemos visto una relación entre este tipo de alumnado y los que cambiaron de modalidad (examen de literatura 
tradicional) por no haber preparado la exposición oral el día que les correspondía. A continuación, mostramos qué modalidad escogieron, según los grupos:

- $1^{\circ}$ de Bachiller B, de 24 estudiantes seis optan por el modelo tradicional y 18 por la exposición oral. De esta muestra dos alumnos tienen problemas de timidez. Los cuatro alumnos restantes no habían planificado su exposición y el mismo día de la presentación optaron por el modelo tradicional.

- $1^{\circ}$ de Bachiller $\mathrm{C}$, de 23 estudiantes dos optan por el modelo tradicional y 21 por la exposición oral. En este caso ambos querían hacer la exposición oral, pero no la prepararon.

- $\quad 1^{\circ}$ de Bachiller $\mathrm{D}$, de 21 estudiantes tres optan por el modelo tradicional y 18 por la exposición oral. En este caso la clase en su totalidad (21 estudiantes) seleccionó la exposición oral, pero (al no haber una preparación previa) cambiaron al formato tradicional de examen.

En cuanto a la estructura de trabajo, el primer día les presentamos los porcentajes de evaluación que íbamos a a seguir y las dos modalidades de trabajo que podían escoger para trabajar la literatura:

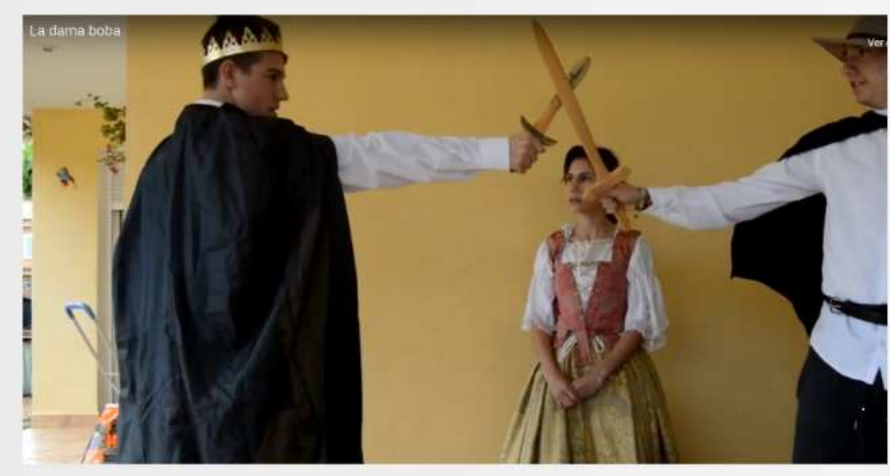

\section{Sistema de evaluación}

- 30\%: Exposiciones (si hay alguna expulsión no podrá optar a esta opción) o examen de literatura oral.

- $10 \%$ : Libro.

- 10\%: Actividades, los comentarios se traerán trabajados de casa para su corrección en clase, en caso contrario perderán un punto.

- $50 \%$ : Examen de comentario literario (30\%) y Sintaxis y morfología (20\%).

- Faltas de ortografía: Se descontará de la nota hasta un punto en el examen siguiendo las pautas PAU, 0,25 puntos por faltas de ortografía y 0,15 cuando sean faltas de acentuación o puntuación. Las faltas ya no se recuperan.

-Que un alumno copie en alguno de los exámenes supone el suspenso inmediato de la evaluación.

- Las fechas de los exámenes sólo se cambiarán si han surgido dudas derivadas del estudio, si por el contrario no se trabaja a un ritmo adecuado la fecha del examen puede adelantarse.

- La calificación definitiva del curso se obtendrá de la media resultante de las tres evaluaciones.

Figura 1: Sistema de evaluación que se siguió en $1^{\circ}$ de Bachillerato B, C y D Fuente: Pareja-Olcina, 2020.

En la programación, esta actividad suponía el 30\% de la nota final de la primera evaluación y el estudiante podía escoger entre la exposición oral o un examen individual sobre los temas expuestos. Para llevar al aula una dinámica de estas características la programación por parte del profesorado es esencial, de forma que el alumnado disponga de una planificación previa con claridad de lo que se le pide, formato y plazos de entrega. En este sentido, el alumnado disponía de las fechas y los temas para su presentación la primera semana septiembre. Esto fue así 


\section{REVISTA INTERNACIONAL DE HUMANIDADES}

porque uno de los retos consistía en realizar la exposición el día elegido. En nuestra trayectoria docente hemos observado la importancia de no movilizar las fechas de exámenes o presentaciones, los resultados del alumnado no mejoran con estos cambios y si saben que existe esta opción suelen buscar un aplazamiento. Sin embargo, el estudiante siempre tuvo la opción de presentarse al modelo de examen individual. Como muestra la gráfica, la mayoría prefirió la exposición oral del tema y la encuesta confirma que un $7 \%$ tenía intención de hacer la presentación.

\section{¿Qué técnica prefieres para realizar el examen de literatura en este nivel? \\ 72 respuestas}

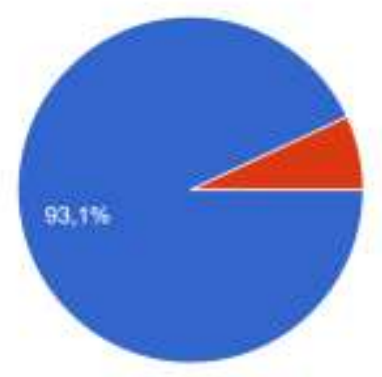

Exposición oral del tema indicado

- Examen de literatura tradicional

Figura 2: Preferencia de los sujetos de estudio sobre modelos de examen

Fuente: Pareja-Olcina, 2020.

\section{¿Has realizado la exposición oral? \\ 72 respuestas}

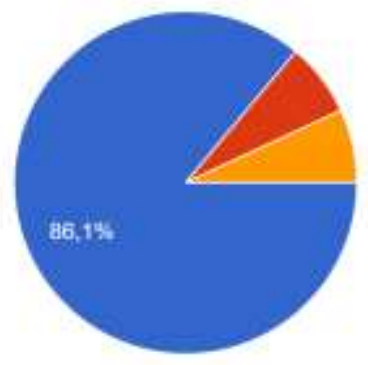

Si

No, pero si que me habia apuntado en un primer momento

No, no me gusta hacer presentaciones orales

Figura 3: Razones de los sujetos de estudio sobre la modalidad de examen escogida Fuente: Pareja-Olcina, 2020.

Algunos discípulos se encontraron con problemas de gestión y organización previa que requiere esta tarea y el día de su presentación cambiaron de modalidad. Las razones que señalan son las siguientes: 
Si no has hecho la exposición de literatura, escoge una de las siguientes razones:

9 respuestos

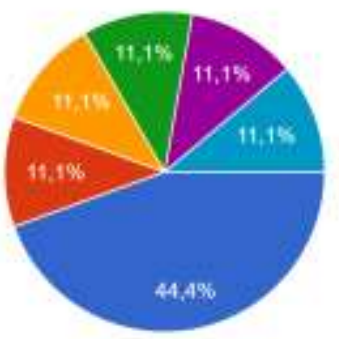

No me he preparado oon antelación la exposiciún, pero me gustaria hacerlo en próximas presentaciones

Pe da verguenza hablat en pública

- No me gustan tos exámenes arales

- Me da un poco de vergìenza pero puedo hacerlo perfectamente

- La nice pero puse otro sutor al que me ponia y no me conto

P La 2 y la 3

Figura 4: Razones de los sujetos de estudio sobre el cambio de modalidad

Fuente: Pareja-Olcina, 2020.

Estos datos demuestran que la propuesta tuvo éxito, pero que algunos estudiantes todavía no dominan las estrategias metacognitivas. El estudiante que se convertía en ponente debía escoger un tema y un día de presentación, entre los ítems que se iban a tratar en la primera evaluación y que estaban dentro del currículum de Lengua Castellana y Literatura para $1^{\circ}$ de Bachiller.

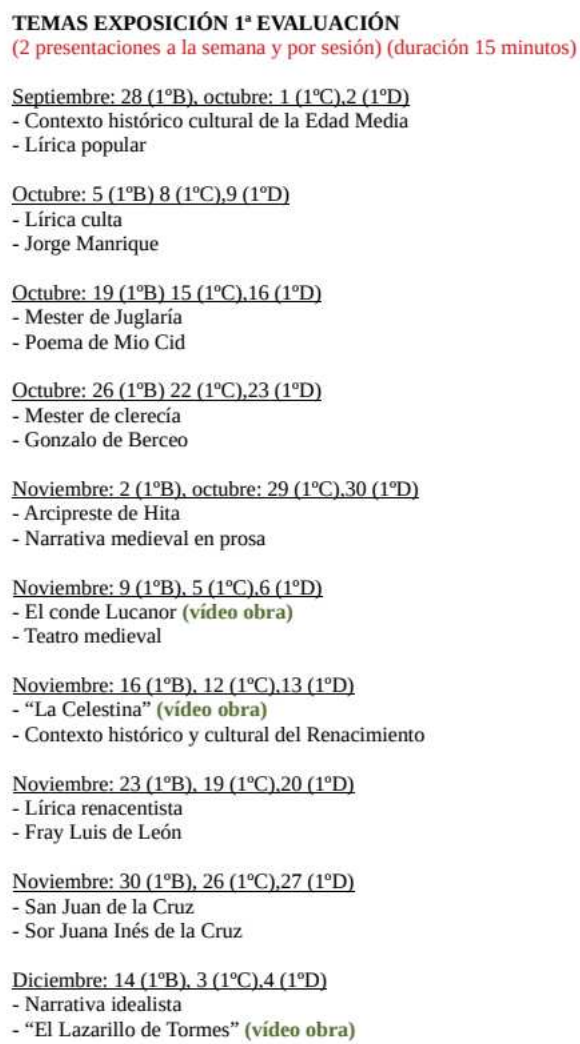

Figura 5: Temas para presentar las ponencias para $1^{\circ}$ de Bachillerato B, C y D

Fuente: Pareja-Olcina, 2020. 
Para presentar algunos temas, los estudiantes tenían la opción de acompañar la presentación de un vídeo de la obra correspondiente de forma que ilustrara el argumento (en la figura anterior está marcado de color verde). En este sentido, nos encontramos con adaptaciones muy interesantes que atrajeron la atención de sus compañeros/as de clase y trabajos que se expusieron en otras clases de primero de bachillerato para asimilar los conceptos ${ }^{3}$.

Para llevar a cabo la presentación, el estudiante debía desarrollar varias habilidades y estrategias que hemos comentado y ajustarse a un límite de tiempo orientativo (15 minutos). En esta comunicación, como la idea era que se convirtieran en profesores, dejaban un margen de tiempo a las preguntas de sus compañeros/as y también podían hacerle preguntas al resto de la clase para comprobar si habían integrado su explicación. En este sentido, destacamos los concursos de preguntas con Kahoot, Genially o, simplemente, levantando la mano en el aula ante las preguntas del presentador. Para preparar este apartado el estudiante contaba con una rúbrica que comentamos al inicio de las presentaciones y que estaba disponible en el Aula Virtual de la asignatura:

\begin{tabular}{|c|c|c|c|c|}
\hline \multirow{2}{*}{\multicolumn{4}{|c|}{$\begin{array}{l}\text { ESTRUCTURA } \\
\text { Número de items } \\
\text { marcados / Puntuación }\end{array}$}} & GRITERIOS DE CALIDAD PARA LA PRESENTACION ORAL \\
\hline & & & & \multirow{4}{*}{$\begin{array}{l}\square \text { La presentación contiene un índice que muestra la estructura que tendrá. } \\
\square \text { En el desarrollo se hace énfasis en los puntos importantes. } \\
\square \text { Contiene unas conclusiones que ayudan a asimilar lo expuesto. } \\
\square \text { En todo momento he sabido en qué parte de la exposición estamos. } \\
\square \text { Las partes de la presentación están bien equilibradas y se han ajustado al tiempo establecido. }\end{array}$} \\
\hline 5 & 43 & 2 & 1 & \\
\hline 2 & 1,5 & 1 & 0,5 & \\
\hline & & & & \\
\hline \multicolumn{4}{|c|}{$\begin{array}{l}\text { CONTENIDO } \\
\text { Número de fterns } \\
\text { marcados / Puntuación }\end{array}$} & \multirow{4}{*}{$\begin{array}{l}\square \text { El orador ha hablado con seguridad sobre el tema, demostrando que lo conoce bien. } \\
\square \text { Ha hecho referencias apropiadas a las fuentes de información. } \\
\square \text { Se han hecho referencias a ejemplos prácticos que me han ayudado a comprender bien lo expuesto. } \\
\square \text { Se ha respondido adecuadamente a todas las preguntas formuladas por la audiencia. }\end{array}$} \\
\hline 4 & 3 & 2 & 1 & \\
\hline 2 & 1,5 & 1 & 0,5 & \\
\hline & & & & \\
\hline \multicolumn{4}{|c|}{$\begin{array}{l}\text { DIAPOSITIVAS } \\
\text { Número de ftems } \\
\text { marcados / Puntuación }\end{array}$} & \multirow{4}{*}{$\begin{array}{l}\square \text { Las diapositivas están numeradas y contienen elementos que permiten saber en qué punto de la } \\
\text { presentación nos encontramos. } \\
\square \text { Cada diapositiva contiene poca letra, frases cortas, y bien visibles. } \\
\square \text { Las fuentes y el tamaño son adecuadas y coherentes. } \\
\square \text { El colorido de la diapositiva resulta agradable a la vista, y el texto contrasta bien con el fondo. } \\
\square \text { Las animaciones ayudan a enfatizar lo importante. }\end{array}$} \\
\hline 5 & $4-3$ & 2 & 1 & \\
\hline 2 & 1,5 & 1 & 0,5 & \\
\hline & & & & \\
\hline \multicolumn{4}{|c|}{$\begin{array}{l}\text { LENGUAJE VERBAL } \\
\text { Y CORPORAL } \\
\text { Número de items } \\
\text { marcados / Puntuación }\end{array}$} & \multirow{4}{*}{$\begin{array}{l}\square \text { El orador ha vocalizado con claridad y ha usado palabras adecuadas a la audiencia (lo he entendido } \\
\text { perfectamente). } \\
\square \text { El volumen y la velocidad al hablar eran adecuados y ha usado alteraciones en el tono para enfatizar } \\
\text { los aspectos importantes. } \\
\square \text { Los gestos corporales han sido moderados (ni excesivamente expresivo ni completamente estático). } \\
\text { Esos gestos se han usado apropiadamente para enfatizar lo importante de la presentación. } \\
\square \text { Ha mirado en general a toda la audiencia, intentando implicarla de esta manera en la presentación. } \\
\square \text { Se nota que han ensayado la presentación previamente. } \\
\square \text { Ha evitado el uso de palabras vagas y de muletillas. }\end{array}$} \\
\hline 6 & $5-4$ & $3-2$ & 1 & \\
\hline 2 & 1,5 & 1 & 0,5 & \\
\hline & & & & \\
\hline \multicolumn{4}{|c|}{$\begin{array}{l}\text { INTERÉS } \\
\text { Número de ftems } \\
\text { marcados / Puntuación }\end{array}$} & \multirow{4}{*}{$\begin{array}{l}\square \text { La presentación ha sabido captar mi interés desde el primer momento. } \\
\square \text { Incluso han usado algunos toques de humor que han aumentado mi motivación. } \\
\square \text { No me he aburrido en ningún momento. } \\
\square \text { He aprendido cosas que no sabía. } \\
\square \text { Han sabido hacer participar a la audiencia. }\end{array}$} \\
\hline s & $4-3$ & 2 & 1 & \\
\hline 2 & 1,5 & 1 & 0,5 & \\
\hline & & & & \\
\hline
\end{tabular}

Figura 7: Criterios de calidad para la presentación oral Fuente: Sansy, 2019. 
Por su parte, el público debía tomar apuntes durante la exposición o hacer algún esquema sobre el tema expuesto. Una vez finalizaba la presentación, destinábamos un tiempo a reflexionar con el grupo-clase los aciertos y los apartados que podría mejorar el ponente en próximas presentaciones. Al hacer al alumnado partícipe de esta reflexión las presentaciones fueron mejorando a medida que avanzaban las semanas.

\section{Resultados}

Muchas de estas exposiciones fueron de gran calidad, dinámicas y oportunas dentro del aula, puesto que los estudiantes se interesaban por la explicación que sus propios compañeros/as les daban. No olvidamos aquí la importancia que tiene el grupo de iguales en este momento de sus vidas. Esto es así porque la adolescencia es un proceso de individualización y desarrollo de la identidad, en el que los jóvenes tratan de alcanzar un determinado nivel de autonomía, reconocimiento y aceptación (Londoño y Valencia, 2010), por esto la integración de contenido, por parte de sus amigos/as, puede tener efectos muy positivos en su aprendizaje. En definitiva, los iguales modelan sus creencias, actitudes y prácticas en esta etapa educativa más que en ningún otro periodo, en atención a lo cual están más predispuestos e interesados en las explicaciones de sus compañeros/as que en las del profesor. Nuestra propuesta consiste en ayudar a los ponentes a perfilar sus presentaciones orales para que los receptores reciban una exposición de calidad por parte de sus compañeros/as.

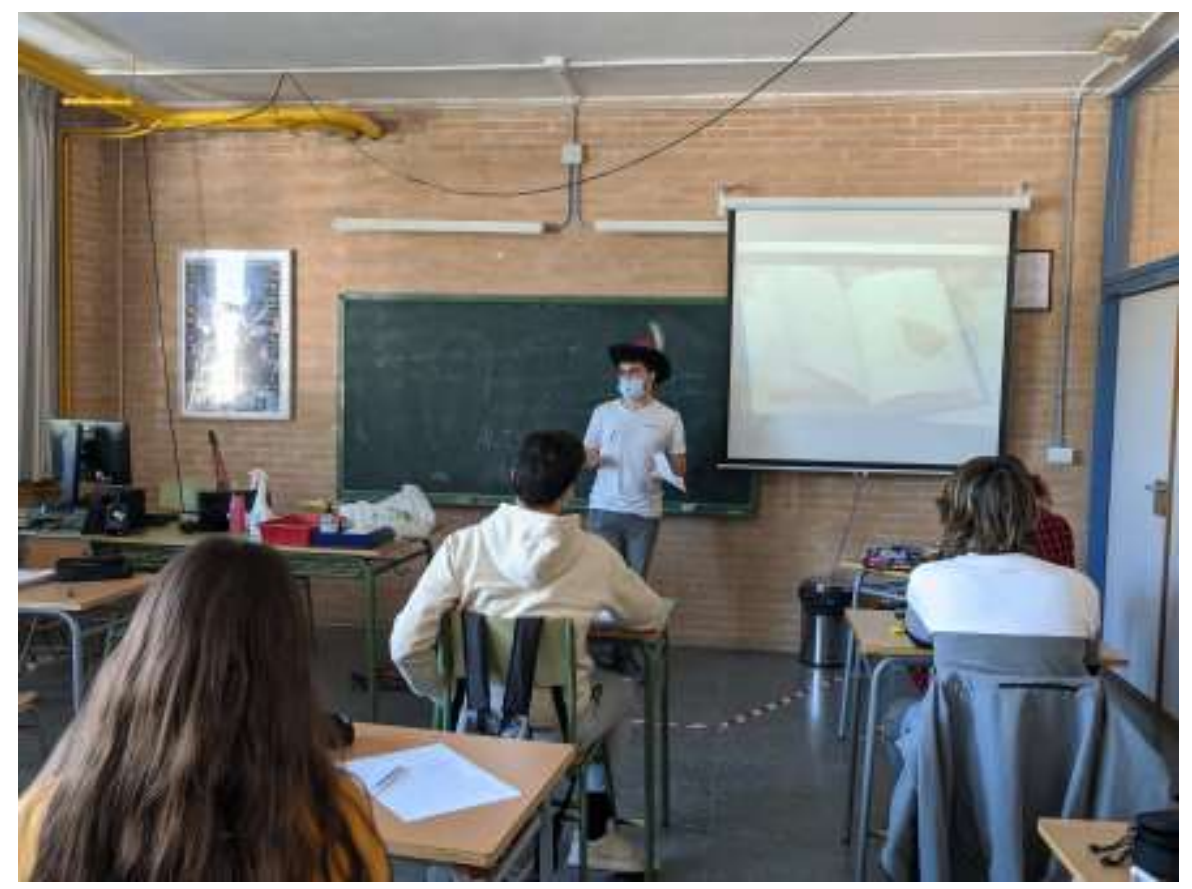

Figura 8: Presentación en clase del tema: Mester de juglaría Fuente: Pareja-Olcina, 2020.

Los datos de la encuesta corroboran que solo el 2`7\% de los estudiantes estaría interesado en realizar exámenes tradicionales, frente al 97,3\% que quieren continuar siendo examinados con esta metodología: 


\section{¿Te gustaria continuar con esta dinàmica de trabajo? \\ 73 respuestas}

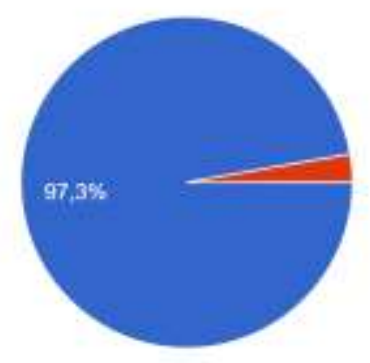

$$
\text { - Si }
$$

Figura 9: Encuesta a los sujetos de estudio sobre su interés por continuar haciendo ponencias a modo de examen

Fuente: Pareja-Olcina, 2020.

Un dato significativo, que se derivó de la entrevista oral, está relacionado con su interés de aplicar esta práctica en otras asignaturas. Referente a eso, la encuesta arrojó los siguientes datos: al 76,7\% de los estudiantes le gustaría incluir esta dinámica en otras asignaturas, el $19,2 \%$ se muestran indecisos y el 4’1\% está en desacuerdo.

\section{¿Te gustaria que otras asignaturas incluyeran esta dinámica de evaluación? 73 respuestas}
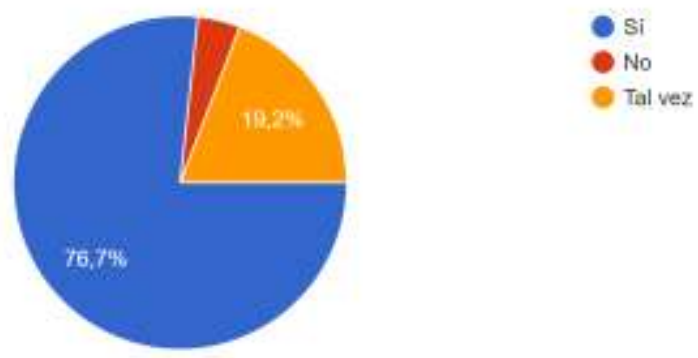

Figura 10: Encuesta a los sujetos de estudio sobre su interés por incluir esta dinámica de examen en otras asignaturas

Fuente: Pareja-Olcina, 2020.

Los resultados académicos de esta propuesta didáctica fueron muy positivos y, en algunos casos, superaron nuestras expectativas. También la propuesta fue muy bien recibida por el alumnado, 57 escogieron la exposición oral, frente a los 11 que decidieron cambiar al examen. En su mayoría, los que tomaron la decisión de cambiar de modalidad fue por una cuestión de habilidades metacognitivas y organización de trabajo. En cuanto a los resultados de la evaluación, se observan calificaciones notablemente más altas en la ponencia que en el examen tradicional.

Uno de los beneficios derivados de esta práctica es que los estudiantes con dificultades de aprendizaje (dislexia y problemas cognitivos), el 5,88\% de la muestra estudiada, supera el examen y el alumno con dislexia obtiene un 10, al no encontrarse con las dificultadas propias de su condición. Esta dinámica es especialmente interesante cuando hablamos de alumnos con trastornos de aprendizaje o discapacidad intelectual, pues responden muy bien a estas dinámicas 
y su integración en el grupo es más eficaz, se favorece su autoestima y pierden el miedo a expresarse. Estos casos deben tenerse en cuenta, ya que la frecuencia del Trastorno por Déficit de Atención e Hiperactividad (TDAH), Dislexia y las Altas Capacidades suman, en conjunto, alrededor de un 24-26\% (La Razón, 2019) y nos dan la clave ante las cifras de fracaso escolar y una larga trayectoria de malos resultados en los diferentes indicadores educativos, como los analizados en el último informe del Programa Internacional para la Evaluación de Estudiantes PISA (2018) ${ }^{4}$. En este sentido, la investigación confirma que adaptarnos a las necesidades de cada estudiante permite acercar de forma efectiva contenido que, visto de otra forma, no atiende las dificultades de aprendizaje. A continuación, aportamos una tabla en la que se puede observar esta diferencia:

Tabla 1: Relación de grupos y calificaciones obtenidas, según su modelo de examen

\begin{tabular}{|c|c|c|c|}
\hline \multicolumn{4}{|c|}{ Exposiciones orales } \\
\hline & $1^{\circ}$ Bach $B$ & $1^{\circ}$ Bac.C & $1^{\circ}$ BacD \\
\hline Aymno 1 & 2,4 & 2,7 & 3,1 \\
\hline Aumno 2 & 3,1 & 2,5 & 3,1 \\
\hline Aมmกo 3 & 2,8 & 3 & 2,5 \\
\hline Asumno 4 & 2,4 & 3. & 2,4 \\
\hline Aymno 5 & 2,8 & 2,3 & 3,1 \\
\hline Aumno 6 & 2.8 & 2,7 & 3,2 \\
\hline Alumno 7 & 1 & 2,5 & 3,2 \\
\hline Aumno 8 & 3 & 3,1 & 3,1 \\
\hline Aumno 9 & 3 & 3 & 2,6 \\
\hline Aumno 10 & 1,5 & 2,6 & 3 \\
\hline Aumino 11 & 3 & 3 & 2,5 \\
\hline Armno 12 & 1,5 & 3 & 2,7 \\
\hline Atumno 13 & 1,8 & 2,3 & $\overline{3,2}$ \\
\hline Atumno 14 & 1,8 & 2.8 & 2,9 \\
\hline Aumno 15 & 0.3 & 3 & 2,6 \\
\hline Atumno 16 & 2,5 & 2,8 & 3 \\
\hline Aumno 17 & 1 & 3 & 1,8 \\
\hline Alumno 18 & 3 & 2,6 & 3 \\
\hline Avimno 19 & & 3,1 & \\
\hline Aumno 20 & & 3 & \\
\hline Aumno 21 & & 2,5 & \\
\hline \#Ponencias & 18 & 21 & 18 \\
\hline Media & 2.21 & 2,79 & 2,83 \\
\hline
\end{tabular}

\begin{tabular}{|l|r|r|r|}
\hline \multicolumn{4}{|c|}{ Examen oral } \\
\hline & 1'Bach.B & 10 Bac.C & 10 Ba.D \\
\hline Aumno 1 & 0 & 1,4 & 1,3 \\
\hline Aumno 2 & 0 & 0,6 & 0 \\
\hline Aumno 3 & 0 & & 0 \\
\hline Aurmno 4 & 0 & & \\
\hline Aumno 5 & 0 & & \\
\hline Aurmno 6 & 0 & & \\
\hline
\end{tabular}

\begin{tabular}{|l|r|r|r|}
\hline PEarmenes & 6 & 2 & 3 \\
\hline Media & 0 & 1 & 0,43 \\
\hline
\end{tabular}

Fuente: Pareja-Olcina, 2020.

\footnotetext{
${ }^{4}$ Los informes de PISA (2018) se pueden consultar en el siguiente enlace: https://www.educacionyfp.gob.es/inee/evaluaciones-internacionales/pisa/pisa-2018/pisa-2018-informes-es.html
} 


\section{REVISTA INTERNACIONAL DE HUMANIDADES}

Por último, nos centraremos en esta metodología como mecanismo para consolidar estrategias de aprendizaje. En el siguiente gráfico los estudiantes encuestados valoran, en primer lugar, la oportunidad de hacer un examen más creativo $(36,4 \%)$. En segundo lugar, reconocen que les ayuda a desarrollar y entrenar una de sus dificultades prioritarias en el ámbito académico: hablar en público (30,3\%). En tercer lugar, apuntan que esta forma de examinarse les permite entender mejor el contenido que presentan $(18,2 \%)$. En la exposición oral reconocieron que de esta forma lo que aprenden dura más en el tiempo y no lo olvidan una vez han finalizado el examen. Por último, un 15,2\% de los encuestados considera que lo más importante de esta evaluación fue la libertad de reflexionar y utilizar la información fruto de su investigación.

\section{¿Que aprendizaje dstacarias de la exposición oral? \\ 66 respuestas}

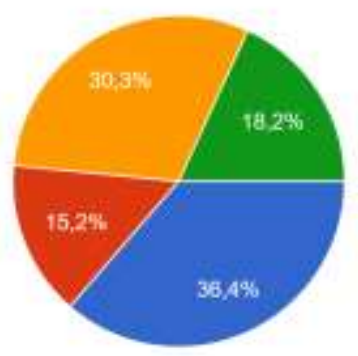

Puede integrar el contenido de forma más creativa que en un examen

- Puedo reflexionar y utlizar la información que yo he investigado

- Me ayuda a desarrollar mis habilidades para hablar en píblico

- Si lo explico lo entiendo mejor

Figura 11: Encuesta a los sujetos de estudio sobre lo que más valoran de esta práctica docente Fuente: Pareja-Olcina, 2020.

Consideramos que esta gráfica nos muestra información relevante sobre las estrategias de aprendizaje y el aporte de esta metodología, por eso también nos pareció oportuno recoger datos en los que los encuestados valoraban cada una de las actividades. En esta modalidad de pregunta recogemos sus valoraciones, donde 1 es poco valorada y 5 muy valorada. En primer lugar, les preguntamos sobre la oportunidad de presentarse a un examen en el que podían expresarse de forma más creativa (presentado vídeos, rapeando las biografías de los autores más destacados, teatralizando las obras literarias, usando disfraces para caracterizar a los escritores seleccionados...). En este sentido el $88,7 \%$ valora de forma muy positiva (entre 4 y 5) esta actividad: 


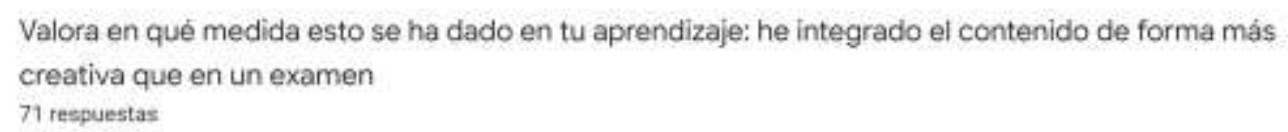

71 respuestas

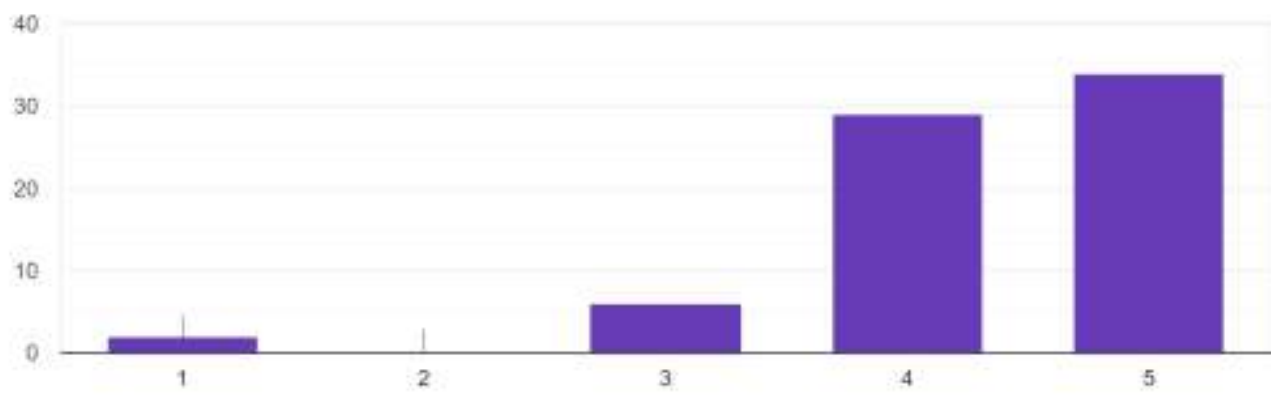

Figura 12: Encuesta a los sujetos de estudio sobre la integración de contenido literario a través de presentaciones Fuente: Pareja-Olcina, 2020.

Un $59,7 \%$ ha valorado de forma muy positiva (5) esta dinámica, porque le ha servido para investigar y reflexionar sobre teoría literaria. Un $25 \%$ lo ha puntuado también de forma positiva (4), mientras que un $11,1 \%$ le da 3 puntos, un $2,8 \%$ dos puntos y un $1,4 \%$ un punto.

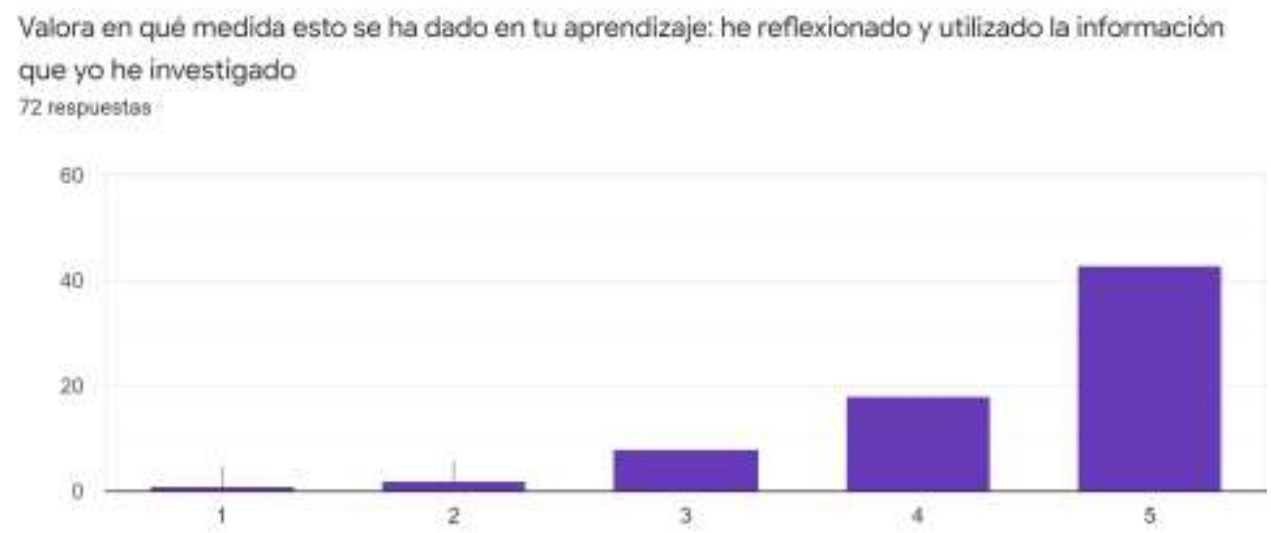

Figura 13: Encuesta a los sujetos de estudio sobre la investigación de contenido literario a través de exposiciones

Fuente: Pareja-Olcina, 2020.

También encontramos valoraciones positivas en cuanto a utilizar esta práctica en el aula para desarrollar habilidades que les permitan hablar en público de forma más segura y fluida. Un $63,4 \%$ le ha dado la máxima puntuación (5), también un 18,3\% lo ha puntuado de forma positiva (4), mientras que un $12,7 \%$ le da 3 puntos, un $2,8 \%$ dos puntos y un $2,8 \%$ un punto. 


\section{REVISTA INTERNACIONAL DE HUMANIDADES}

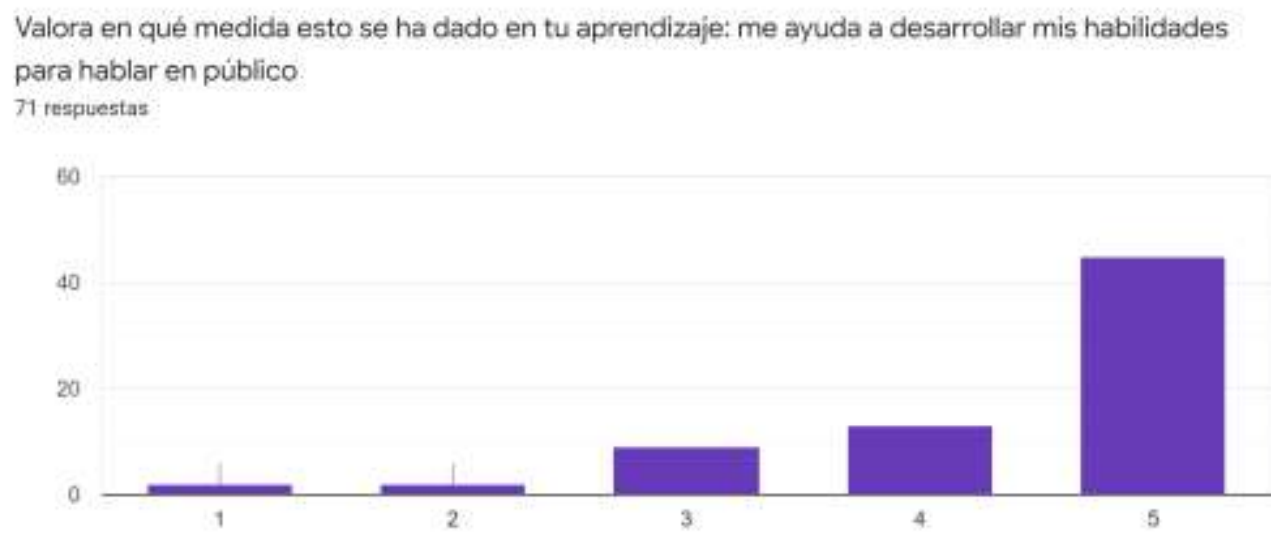

Figura 14: Encuesta a los sujetos de estudio sobre habilidades para hablar en público a través de exposiciones orales Fuente: Pareja-Olcina, 2020.

Por último, la mayoría de los estudiantes encuestados valida la teoría que habíamos apuntado en la introducción: se comprende y entiende mejor lo que somos capaces de explicar. Un 47,9 \% le ha dado la máxima puntuación (5), también un 32,4\% lo ha puntuado de forma positiva (4), mientras que un $16,9 \%$ le da 3 puntos y un $2,8 \%$ un punto.

\section{Valora en qué medida esto se ha dado en tu aprendizaje: si lo explico lo entiendo mejor 71 respuestas}

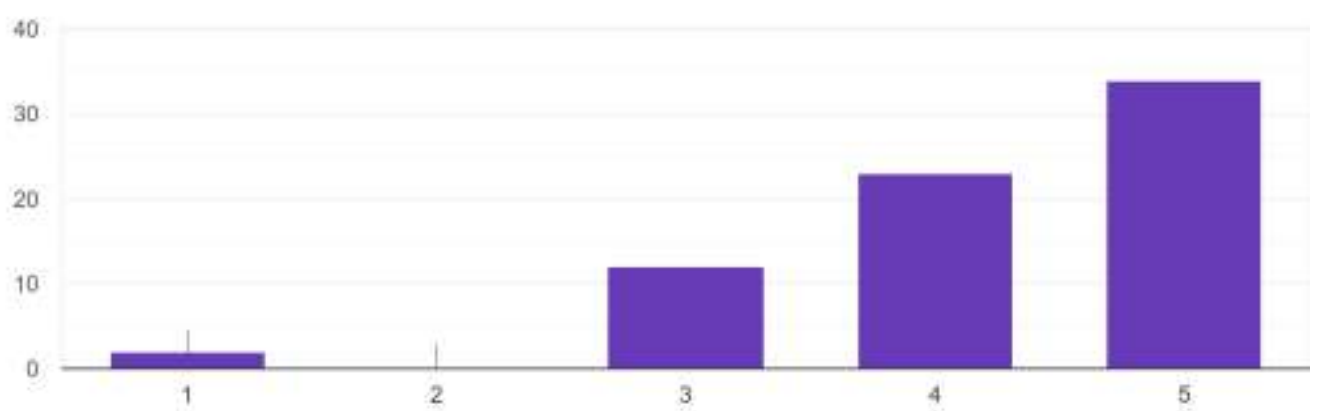

Figura 15: Encuesta a los sujetos de estudio sobre usar las ponencias para comprender el contenido literario Fuente: Pareja-Olcina, 2020.

\section{Conclusiones}

Aprender a hacer una exposición oral es un proceso complejo que requiere preparación, planificación, análisis de datos, dominar recursos linguiísticos, fomentar la atención y la escucha atenta. Creemos que los estudiantes deben encontrar un espacio para expresarse oralmente en un contexto académico y el aula de Lengua Castellana y Literatura es el lugar idóneo para desarrollar estas habilidades. Para ello hemos repasado las estrategias de trabajo más relevantes 
en el marco académico y hemos visto que el éxito depende de varios factores directos e indirectos que se centran en el dominio de recursos metacognitivos, sociales, emocionales y comunicativos.

La formación de ciudadanos competentes en esta etapa de sus vidas constituye un reto para nuestro sistema educativo. La gramática, la literatura y la organización estructural de sus ideas sientan la base para continuar con su aprendizaje. La teoría curricular que se pretende enseñar en este nivel debe conectar con las necesidades e inquietudes de estos jóvenes, por este motivo en la propuesta metodológica los comunicadores utilizaban herramientas que les eran propias (programas de edición de vídeo, música, rap, herramientas TIC, videojuegos, concursos literarios...) y que, además, favorecían una participación activa, motivada y productiva de los asistentes.

La aplicación de este modelo investigación-acción creemos que puede ser útil para diseñar propuestas de mejora en la programación del aula. Las calificaciones que han obtenido y el contraste con los que optaron por un modelo de examen tradicional han demostrado que es una herramienta eficaz para integrar los contenidos, trabajar técnicas de aprendizaje autónomo y mejorar la competencia oral de nuestro alumnado en entornos formales. Todas ellas características esenciales para desenvolverse en la sociedad de siglo XXI. Además, la encuesta ha verificado que el alumnado se siente más atraído hacia estas metodologías y, por tanto, más motivado.

Finalmente, queremos destacar que este trabajo ha incluido dos facetas en el aula. Por un lado, se ha tenido en cuenta la exposición oral del ponente y, por otro, la escucha atenta del resto de la clase. De forma que el alcance del proyecto también abarca al resto del alumnado, puesto que se beneficia de esta herramienta educativa. La audiencia tenía que hacer esquemas, dibujos sobre la exposición, que le permitieran recordar y comprender los temas expuestos por sus compañeros/as de aula. Así se trabajan las dos vertientes claves de la comunicación: aprender a comunicarse y aprender a escuchar.

En definitiva, esta propuesta metodológica confirma el potencial educativo de aumentar las exposiciones orales en detrimento de los exámenes memorísticos, en un nivel concreto educativo ( $1^{\circ}$ de Bachillerato) y en una asignatura instrumental del sistema educativo español: Lengua Castellana y Literatura. Sin embargo, los resultados son aplicables a otras materias y prácticas académicas de otros países (Brooks et al., 2014; Nor et al., 2014; Villar, 2011), ya que el resultado final de aplicar esta herramienta didáctica en el aula trae consigo la mejora de las estrategias de aprendizaje, la competencia comunicativa, la motivación en el aula, la adaptación curricular de alumnos con dificultades de aprendizaje y les permite aprender a aprender.

\section{Agradecimientos}

Me gustaría agradecer la colaboración de todos los adolescentes que han formado parte de este proyecto y que siguen motivándonos para encontrar nuevas herramientas educativas, que nos permitan acompañarlos y prepararlos en esta nueva era. Y, muy especialmente, a todos aquellos docentes que me han aportado tanta sabiduría y comprensión en mi carrera como aprendiz, especialmente a Társila, la maestra que creyó en mí y en mi potencial como lectora.

\section{REFERENCIAS}

Alonso, C., Gallego, D. y Honey, P. 1997. Los Estilos de Aprendizaje. Bilbao: Mensajero.

Alonso, C. M. y Gallego, D.J. 2003. Cómo diagnosticar y mejorar los estilos de aprendizaje. Madrid: UNED. 
Brooks, G., y Wilson, J. 2014. "Using oral presentations to improve students' English language skills". Kwansei Gakuin University Humanities Review, 19(1): 199-212.

Candy, P. C. 1991.Self-Direction for Lifelong Learning. A Comprehensive Guide to Theory and Practice. San Francisco: Jossey-Bass.

Castejón, J.L., Montanes, J. y García Correa, A. 1993. "Estrategias de aprendizaje y rendimiento académico". Revista de Psicología de la Educación, 13: 89-105.

Chow, A. F., Woodford, K. C., y Maes, J. 2011. "Deal or No Deal: using games to improve student learning, retention and decision-making". International journal of mathematical education in science and technology, 42(2): 259-264.

Derry, S. y Murphy, D. A. 1986. "Designing systems that train learning ability". Review of Educational Research, 56: 1-24.

Dehaene, S. 2020. How we learn: Why brains learn better than any machine... for now. Penguin.

Dunlosky, J., Rawson, K. A., Marsh, E. J., Nathan, M. J., y Willingham, D. T. 2013. "Improving students' learning with effective learning techniques: Promising directions from cognitive and educational psychology". Psychological Science in the Public Interest, 14(1): 4-58.

European Commission. 2020. Communication from the Commission to the European parliament, the council, the european economic and social committee and the committee of the regions. Recuperado de https://ec.europa.eu/education/sites/default/files/document-library-docs/eeacommunication-sept2020_en.pdf

Esteban, M., Ruiz, C. y Cerezo, F. 1996. "Validación del cuestionario ILPR, versión española". Anales de Psicología, 12: 133-151.

Fernández, S. 2004. "Las estrategias de aprendizaje". Vademécum para la formación de profesores, Madrid: SGL.

Gràcia, M., Galván-Bovaira, M. J., y Sánchez-Cano, M. 2017. “Análisis de las líneas de investigación y actuación en la enseñanza y el aprendizaje del lenguaje oral en contexto escolar". Revista Española de Lingüística Aplicadal, 30(1): 188-209.

Gregorc, A.F. 1979. "Learning/teaching styles: potent forces behind them". Educational Leadership, 36: 234-237.

Gregorc, A.F. 1982. ORGANON: an adult's guide to style. Columbia: Gregorc Associates Inc.

Gregorc, A. F. 2002. "Frequently asked questions on style". Retrieved July, 8, 2007.

Hewitt, P. L., Habke, A. M., Lee-Baggley, D. L., Sherry, S. B., y Flett, G. L. 2008. "The impact of perfectionistic self-presentation on the cognitive, affective, and physiological experience of a clinical interview". Psychiatry: Interpersonal and Biological Processes, 71(2): 93-122.

Hunt, D.E. 1979. "Learning style and student needs: an introduction to conceptual level". Students learning styles: diagnosing and prescribing preograms. Virginia: National Association of Secundary School Principals: 27-38.

Jandrić, P., y Hayes, S. 2020. "Postdigital we-learn". Studies in Philosophy and Education, 39(3): 285-297.

Jovellanos, G. M. 2012. Memoria sobre educacion publica. Madrid: Biblioteca Nueva.

Kennedy, M. M. 2019. "How we learn about teacher learning". Review of research in education, 43(1): 138-162.

La Razón. 2019, 15 diciembre. El porcentaje de fracaso escolar se mantiene en cifras descontroladas. La Razón. Recuperado de https://www.larazon.es/familia/20191215/ylrwqkcr4zagtjcu57rfskqdza.html

Londoño, C. y Valencia, S. C. 2010. "Resistencia de la presión de grupo, creencias acerca del consumo y consumo de alcohol en universitarios". Anales de Psicología, 26 (1): 2733. 
Monereo, C. y Castello, C. 1997. Estrategias de aprendizaje: cómo incorporarlas a la práctica educativa. Barcelona: Edebé.

McGuire, S. Y. 2020. "Help Students Improve Their Learning by Transforming Their Attitudes About the Meaning of learning". Journal of Transformative Learning.

Navarro Jiménez, M. J. 2008. Cómo diagnosticar y mejorar los estilos de aprendizaje. Almería: Asociación Procompal.

Nor, H. N. H. M., y Shahrill, M. (2014, June). "Incorporating the use of poster and oral presentations as an alternative assessment in the teaching of secondary mathematics". En Proceedings of the 2nd International Conference on Social Sciences Research: 369-378.

Keefe, J.W. 1988. Profiling and utilizing learning style. Virginia: National Association of Secundary School Principals.

Kolb, D.A. 1984. Experiential learning experiencie as the source of learning and development. Englewood Cliffs, New Jersey: Prentice-Hall.

Osses Bustingorry, S., y Jaramillo Mora, S. 2008. "Metacognición: un camino para aprender a aprender”.Estudios pedagógicos (Valdivia), 34 (1): 187-197.

Oxford, R. L. 1990. Language Learning Strategies: What Every Teacher Should Know. Boston: Heinle y Heinle.

Renninger, K. A., y Hidi, S. 2015. The power of interest for motivation and engagement. Routledge.

Salmerón, H. y Gutierrez-Braojos, C. 2009. "Desarrollo de la Competencia Matemática a través de programas de Aprender a Aprender infusionados en el currículum ordinario". Revista Iberoamericana de Evaluación Educativa, 2 (2): 141-156.

Salmerón, Gutierrez-Braojos, Rodríguez-Fernandez y Salmeron-Vilchez. 2010. "Influencia del aprendizaje cooperativo en el desarrollo de la competencia para aprender a aprender en la infancia”. Revista Española de Orientación y Psicopedagogía, 21: 308-319.

Salmerón, H., Gutierrez-Braojos, C., Rodriguez, S., y Salmeron-Vilchez, P. 2011. "Metas de logro, estrategias de regulación y rendimiento académico en diferentes estudios universitarios". Revista de Investigación Educativa, 29(2): 467-477.

Salmerón, H., Gutierrez-Braojos, C., Fernández-Cano, A. y Salmeron-Vilchez, P. 2010. "Aprendizaje autorregulado, creencias de autoeficacia y desempeño en la segunda infancia." RELIEVE, 16(2): 1-18.

Schmeck, R. 1982. Inventory of Learning Processes. Students Learning Styles and Brain Behaivor. Ann Arbor, Michigan: ERIC.

Schmeck, R. 1988. Learning Strategies and Learning Styles. New York: Prenum Press.

Shi, H. 2017. "Learning strategies and classification in education". Institute for Learning Styles Journal, 1(1): 24-36.

Stake, R. E. 1998. Investigación con estudio de casos. Madrid: Morata.

Villar, C. M. 2011. "Las presentaciones académicas orales de los estudiantes alemanes de E/LE”. Revista Nebrija de Lingüística aplicada a la enseñanza de Lenguas, (10): 130172.

Walker, R. 2002. "Case study, case records and multimedia". Cambridge Journal of Education, 32: 109-127.

Weinstein, C.E. y Danserau, V. C. 1985. "Learnig strategies: the how of learning”. En J. W. Segal y otros, Thinking and learning skills. Hillsdale: Erlbaum. 


\section{SOBRE LA AUTORA}

María Pareja-Olcina: Profesora asociada, Departamento de Filología y Cultura Europeas, Área Español, Universitat Jaume I, Castellón, España. Profesora asociada de la Universitat Jaume I de Castellón en el Departamento de Filología Hispánica, doctora en Lengua y Literatura Hispánicas (2010) con la calificación de apto cum laude por unanimidad y premio extraordinario. Licenciada en Ciencias de la Información (2004). Tanto en la Universidad Jaume I como en Secundaria, tutoriza (desde 2017) estudiantes del Máster en Profesor de Educación Secundaria en la especialidad de Lengua Castellana y Literatura. 
La Revista Internacional de Humanidades proporciona un espacio para el diálogo y la publicación de nuevos conocimientos en el seno de las humanidades que se sustentan sobre tradiciones pasadas al tiempo que permiten establecer un programa renovado para un futuro que incorpore además la transformación digital de estos saberes. Las humanidades son un ámbito de aprendizaje, reflexión y acción, y un lugar de diálogo entre distintas epistemologías, perspectivas y áreas de conocimiento. En estos inestables lugares de entrecruzamiento del saber humano, las humanidades podrían ser capaces de neutralizar la estrechez de miras de los modernos sistemas de conocimiento.

Los artículos de la revista abarcan un terreno muy amplio, desde lo general y especulativo hasta lo particular y empírico. No obstante, su preocupación principal es redefinir nuestra comprensión de lo humano y mostrar diversas prácticas disciplinarias dentro de las humanidades. En un momento en que las tendencias teóricas dominantes parecen confluir en políticas que a menudo conducen a la humanidad a situaciones intelectuales y sociales poco

satisfactorias, esta revista pretende reabrir el debate acerca de las diversas facetas de los seres humanos tanto por razones prácticas como teóricas.
La revista es relevante para los académicos e investigadores provenientes de un amplio espectro de disciplinas dentro de las humanidades, para los profesores universitarios y los educadores, así como para cualquier persona con interés e inquietud por las humanidades.

La Revista Internacional de Humanidades es una revista académica sujeta a revisión por pares. 